\title{
Design of a novel 6-DOF planar maglev system
}

\author{
Yi-Chih Lai*, Jia-Yush Yen \\ Department of Mechanical Engineering, National Taiwan University, No. 1, Sec. 4, Roosevelt Road, Taipei 106, Taiwan, ROC
}

Available online 2 March 2006

\begin{abstract}
This paper describes a novel single-deck and six degree-of-freedom (DOF) planar maglev positioning system design. The proposed design uses an array of solenoids to levitate and to hold a permanent magnet carrier in place. The solenoids are excited separately to generate restoring forces when the permanent magnet carrier is displaced from its equilibrium position. The research uses the ANSOFT finite element analysis simulation to analyze the solenoid restoring forces and to motivate a suitable permanent magnets arrangement. Active control on the solenoid currents is then used to maintain the carrier position. The system identification is carried out by perturbing the experimental set-up from its equilibrium position. The simulation results based on the identification models show that simple control is effective for maintaining the carrier position. Initial implementation has also showed that the concept is feasible.
\end{abstract}

(C) 2006 Elsevier B.V. All rights reserved.

Keywords: Permanent magnets; Planar maglev; Solenoids

\section{Introduction}

The maglev motion guide ways are usually designed either for very short moving range or for long-range movement with one moving DOF. To achieve long-range motion or higher DOF motion, stacked design is usually necessary. The novel system in this article, on the other hand, uses a single-deck planar design. The maglev system consists of an array of fixed electromagnetic solenoid stators and a permanent magnet carrier stage. The system excites the solenoids to drive and to position the permanent magnet carrier stage. The design is less complicated than the other single-deck planar maglev systems [1-3]. The analysis in this research is based on the ANSOFT FEA software. The 3D simulation results allow one to investigate the solenoid effects and to reach at a solenoid excitation configuration that achieves restoring forces for the permanent magnet carrier.

Because of the naturally unstable structure of the platform, the system requires active solenoid current control to achieve simultaneously stable carrier levitation and positioning. There is an experimental set-up built for system verification, and system identification procedures

\footnotetext{
${ }^{*}$ Corresponding author. Tel.: + 886233664508 .

E-mail address: d89522006@ntu.edu.tw (Y.-C. Lai).
}

are carried out to derive a servo control model. Simulation results show that proper feedback control action can achieve stable positioning in all six axes. Initial implementation has also confirmed the concept.

\section{Maglev system design}

The goal of the analysis is to reach a configuration that can provide restoring forces to the carrier in all manners of carrier attitude deviation. The scales of the cylindrical solenoids in the system are $20 \mathrm{~mm}$ in the outside diameter; $10 \mathrm{~mm}$ in the inside diameter; $10 \mathrm{~mm}$ in height and 370 turns. The material for the permanent magnet is $\mathrm{NdFeB}$. Its diameter is $10 \mathrm{~mm}$, remanence is $1.29 \mathrm{~T}$ and coercivity is $990 \mathrm{kA} / \mathrm{m}$.

\subsection{Analysis of simulation results}

Fig. 1 shows the 3D mimic framework of the magnets arrangement. The simulation tasks take the center of the solenoid, passing $1 \mathrm{~A}$ current, as the origin and move the horizontally placed circular magnet along the $x$ direction. Fig. 2 shows the $x$ and $z$ direction force variations while keeping the magnet at different air gap altitudes. The repulsion $(\mathrm{Fx})$ reaches maximum at $\pm 6 \mathrm{~mm}$ and zero at $0 \mathrm{~mm}$. The buoyancy $(\mathrm{Fz})$ reaches its maximum at the 
center of the solenoid. Fig. 2 shows that there maintains enough buoyancy force to elevate the magnet. The force altitude relationship from the simulations does not exhibit a concave characteristic; therefore, some active control may be necessary.

\subsection{Design of the 6-DOF planar maglev system}

Based on the simulation results, the proposed design assembly provides a restoring force, by placing the magnet between two solenoids, to move the carrier back from any position deviations and a levitation force, by placing the magnet upon the solenoid, to counteract the carrier gravity. From the analysis, a novel single-deck planar maglev system is proposed as shown in Fig. 3. The maglev platform, $140 \times 140 \mathrm{~mm}^{2}$, has 29 circular magnets with 37 solenoids to form the array. Fig. 4a shows the five horizontal magnets designed to provide buoyancy force and to control the carrier altitude. The analysis results suggested the design in Figs. 4(b) and (c) to achieve $x$ and $y$ direction motion. Respectively, there are eight horizontal magnets controlled by 12 solenoids (4 rows) to exert restoring forces to the carrier. The corresponding forces generated by the magnets are also shown in the figures. When yaw disturbance occurs as in Fig. 4d, the four

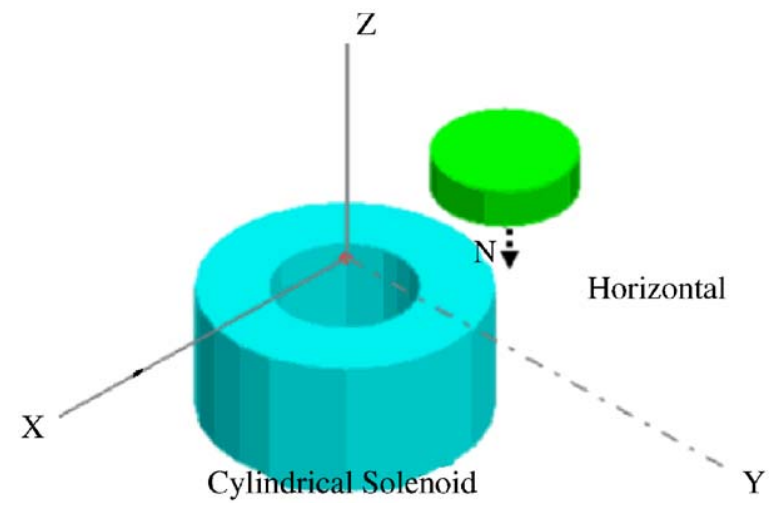

Fig. 1. A 3D mimic framework of the circular magnet and solenoid. bottom solenoids will be excited with different currents to provide restoring forces to the yaw magnets. The roll control is similar as shown in Fig. 4e and pitch control as shown in Fig. 4f. Thus, one is able to control all 6-DOF of the maglev carrier stage.

\section{System identification and control}

Fig. 5 shows the prototype experimental system with six sensors. There are four LED displacement sensors and two eddy current displacement sensors to detect the 6-DOF movements. The system is thus a six-input and six-output system. Because of the very complicated system configuration, the servo design adopts a straight forward approach to treat each channel as independent single-input singleoutput sub-system.

This research carries out the system identification by separately perturbing the coil currents that control the motion axes and measuring off the movements of the corresponding axes signals. The system ID procedure is based upon the autoregressive with exogenous input

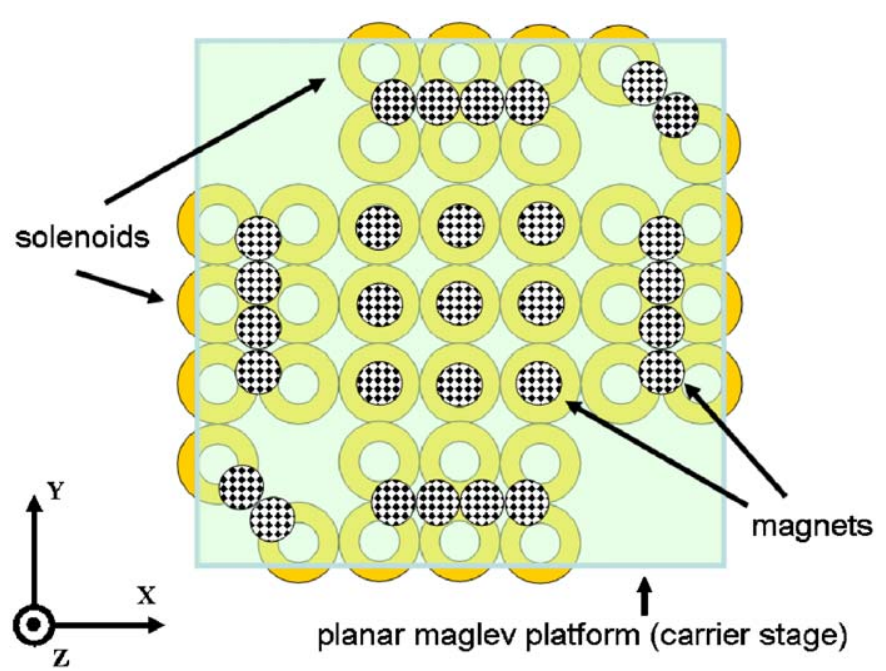

Fig. 3. Design structure of the novel planar maglev system.
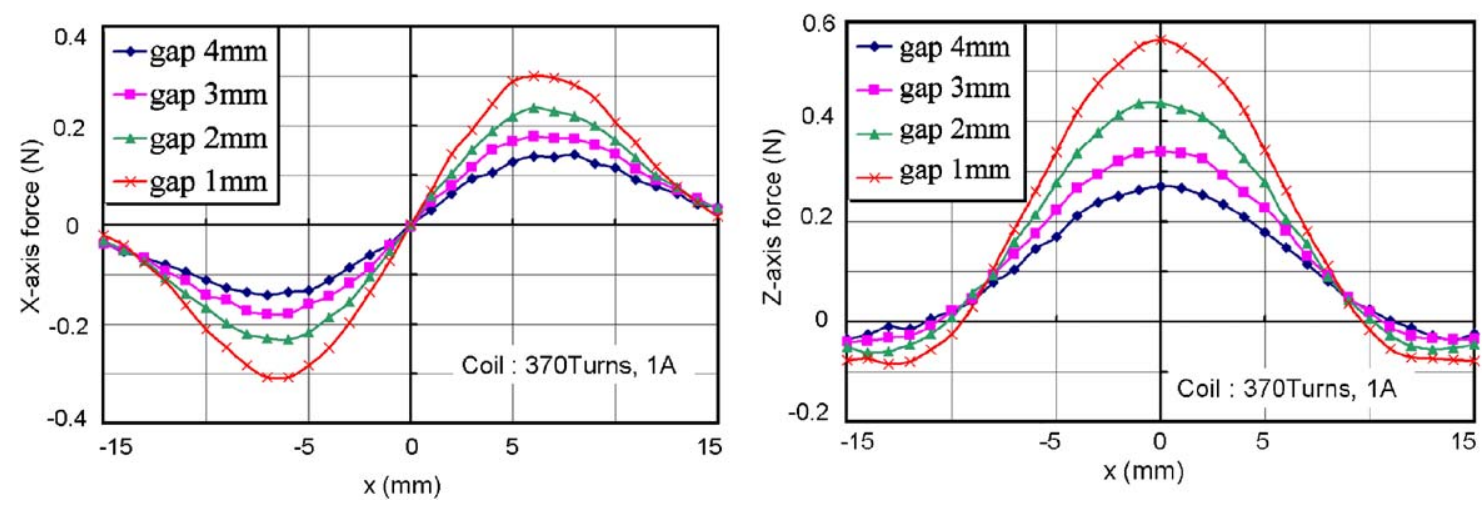

Fig. 2. $X$-axis and $Z$-axis force of the magnet at different air gaps. 


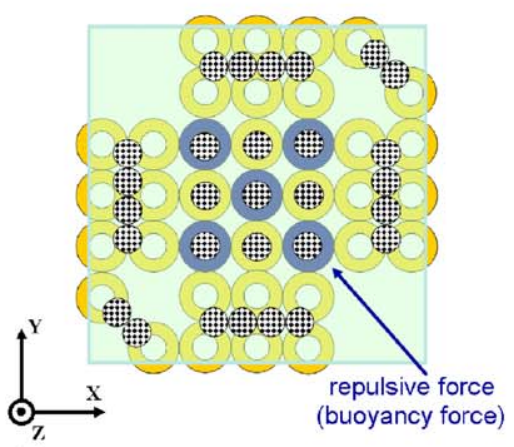

(a)

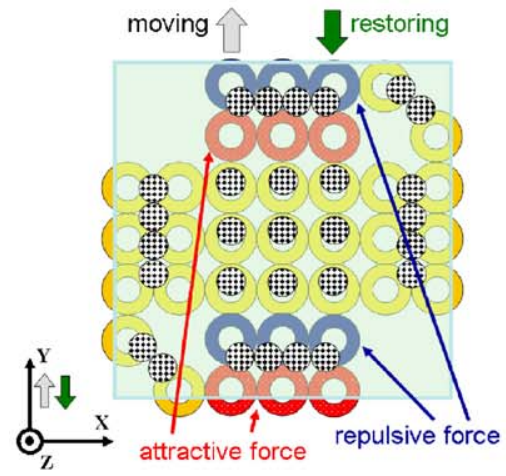

(c)

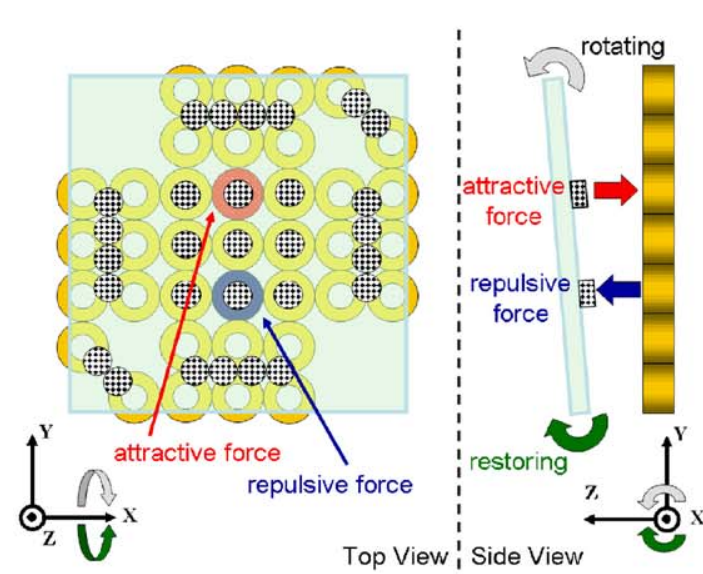

(e)

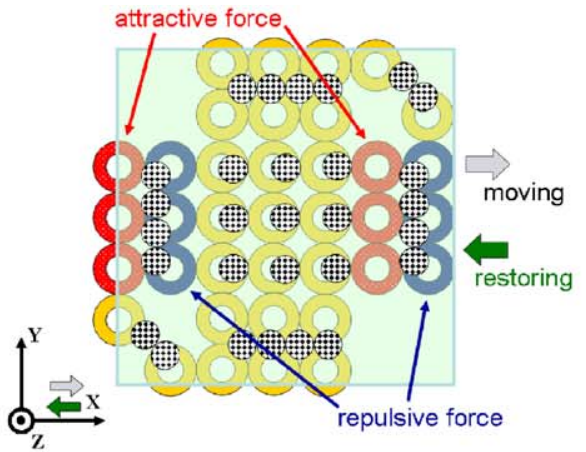

(b)

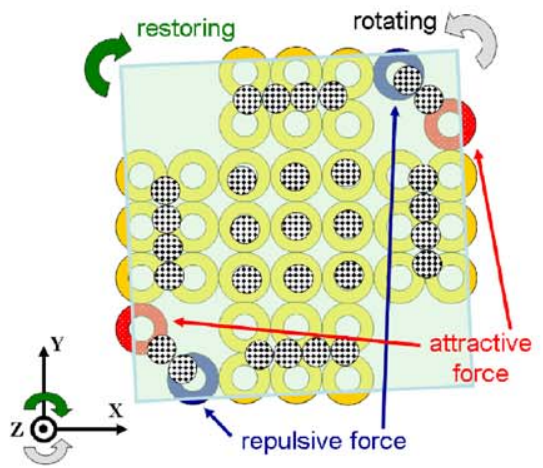

(d)

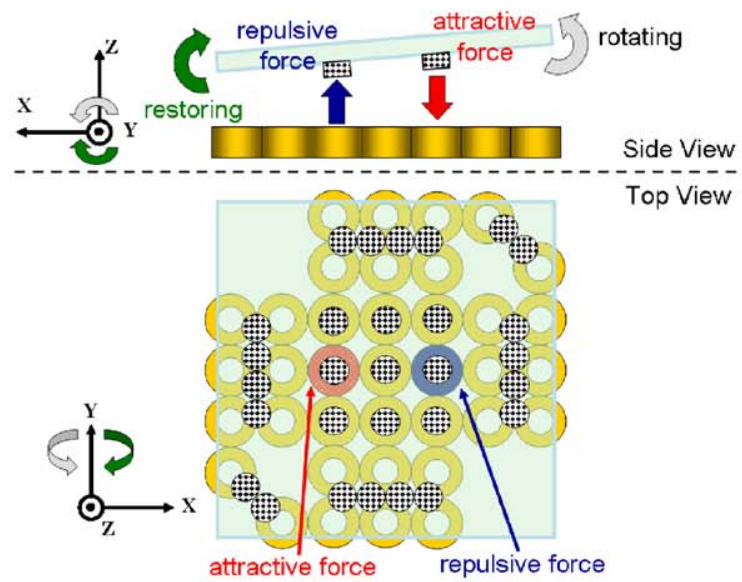

(f)

Fig. 4. A 6-DOF driver of the novel planar maglev system: (a) $Z$-direction, (b) $X$-direction, (c) $Y$-direction, (d) $Z$-axis rotation, (e) $X$-axis rotation, and (f) $Y$-axis rotation.
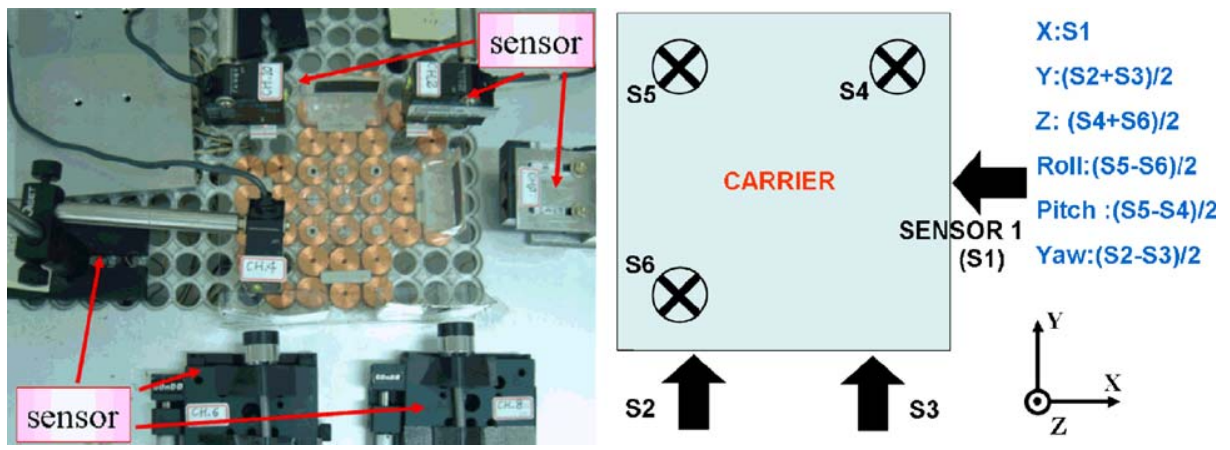

Fig. 5. Prototype of the maglev system with six sensors. 

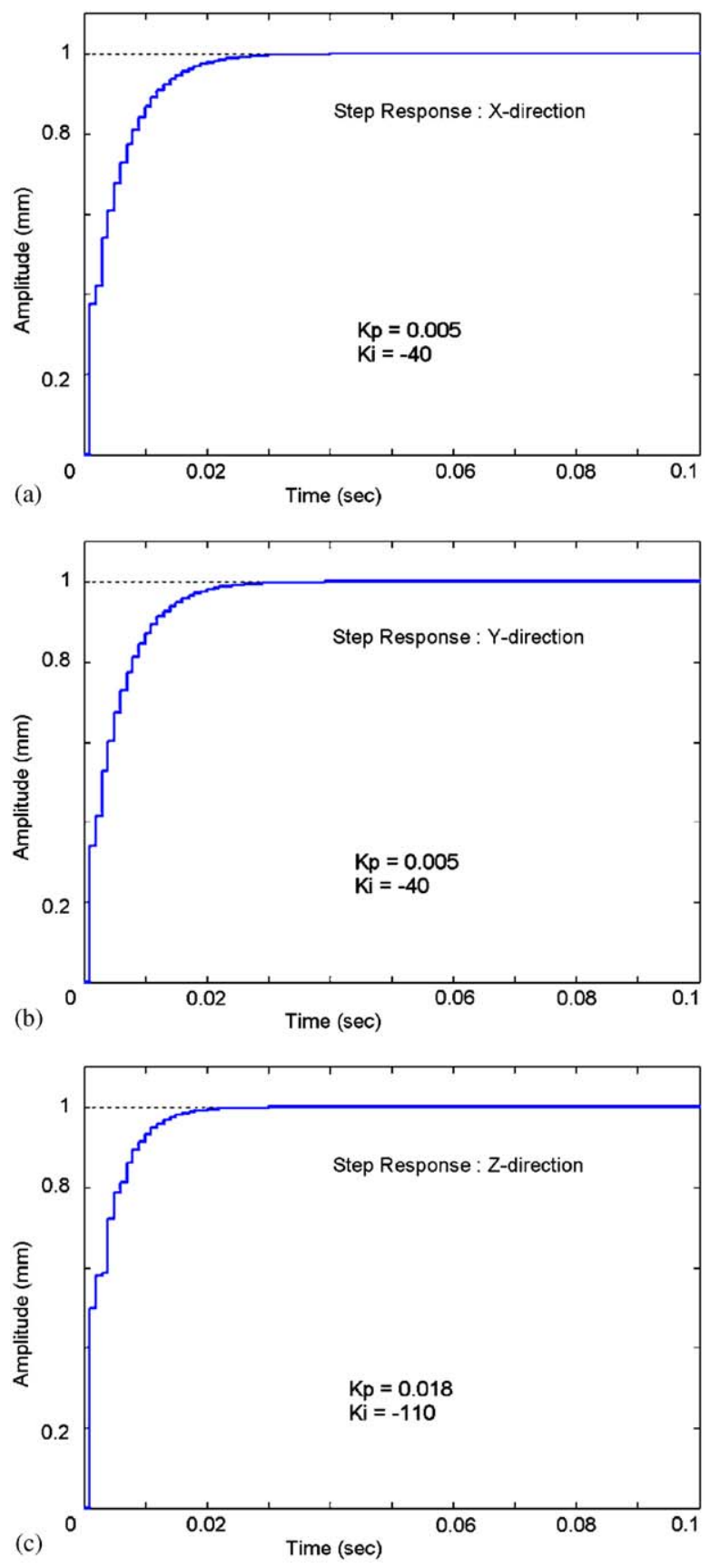

Fig. 6. PI controller's step response: (a) $X$-direction, (b) $Y$-direction, and (c) $Z$-direction.

(ARX) model and the identification procedure resulted in 6 second-order system models for the axes motions. The dynamic system simulations are carried out based on the identification models. Unlike what was expected, the models consist of rather simple structure, and simple PIcontrol seem to suffice. Fig. 6 shows the resulted system responses on $x, y$ and $z$ direction. The system identification data was taken at $1 \mathrm{kHz}$ sampling rate. The control responses do not show any overshoot, and the signals converge quickly. This indicates that the active feedback control would be effective and achieve stable positioning on the carrier. Fig. 7 shows the initial implementation
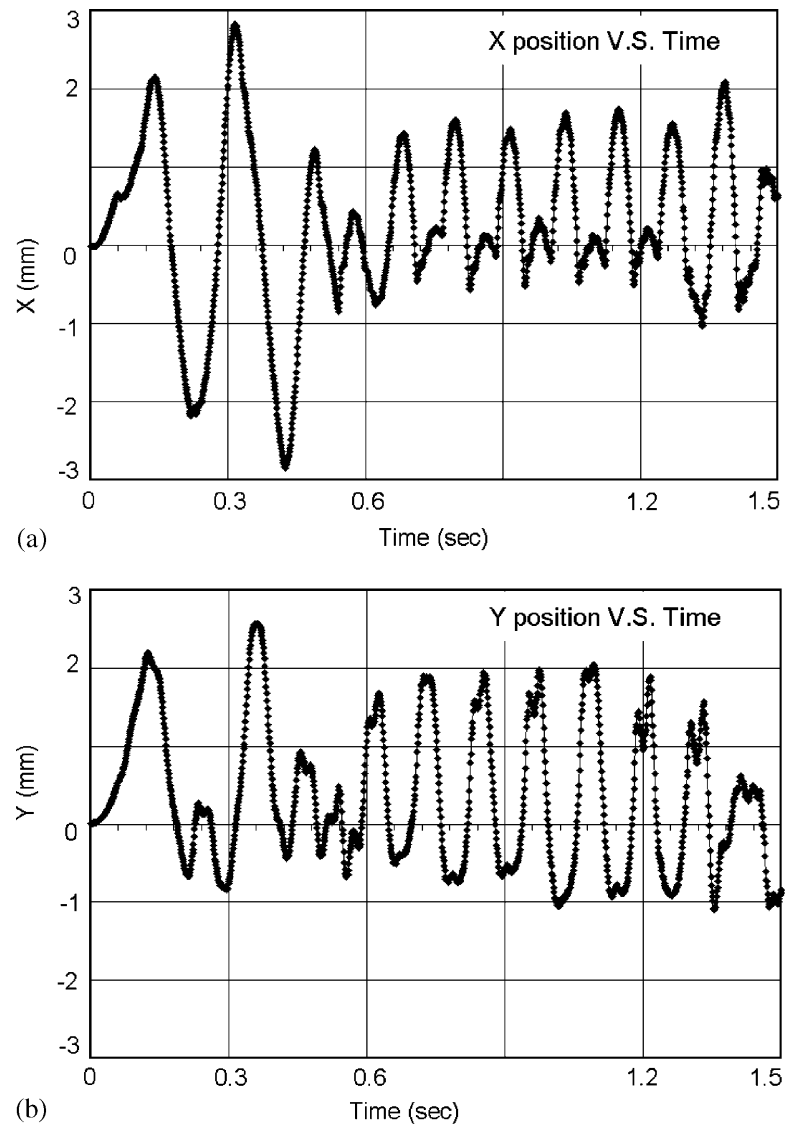

(b)

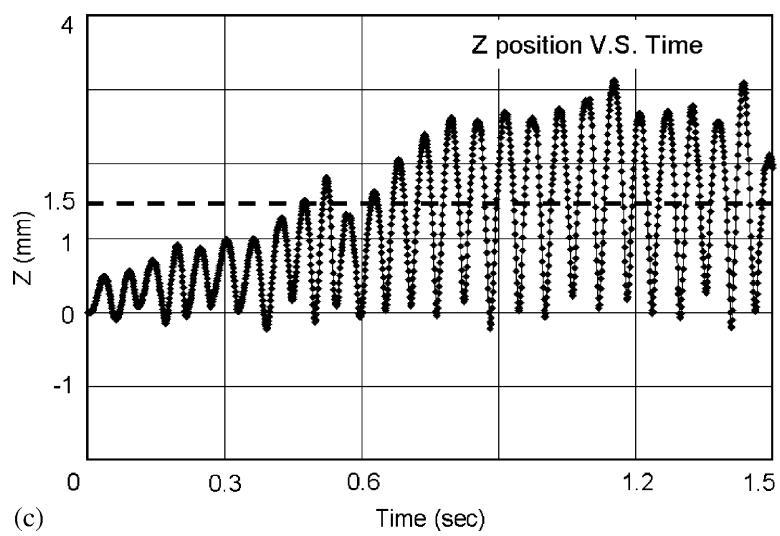

Fig. 7. Implementation results: (a) $X$-direction, (b) $Y$-direction, and (c) $Z$ direction.

results. The responses in all three directions are all highly oscillatory. There is an oscillation of magnitude reaching $1.5 \mathrm{~mm}$ in the $z$ direction. There are still a lot of rooms for further servo tuning; however, these results clearly indicate that the controller is able to hold the stage in place. Due to space limit, the pitch, roll and yaw responses are not shown.

\section{Conclusions}

By investigating the FEA analysis results, it is possible to construct a solenoid-controlled pattern in the planar 
maglev system to provide the moving carrier restoring forces. The system then requires active feedback control on the solenoid current. A test set-up enables the research to perform system identification. Six separate SISO models are identified for the controller design. The control responses on the system identification models show that active control on the solenoid model is effective, and implementation results confirm the feasibility of the concept.

\section{References}

[1] A.F. Flores Filho, A.A. Susin, M.A. da Silveira, IEEE Trans. Magn. 35 (5) (1999) 4034.

[2] H.S. Cho, C.H. Im, H.K. Jung, IEEE Trans. Magn. 37 (5) (2001) 3762.

[3] K.S. Jung, Y.S. Baek, IEEE Trans. Magn. 38 (3) (2002) 1539. 